\title{
The impact of smoking in primary spontaneous pneumothorax
}

\author{
Yeung-Leung Cheng, MD, PhD, ${ }^{a}$ Tsai-Wang Huang, MD, ${ }^{a}$ Chih-Kung Lin, MD, ${ }^{\mathrm{b}}$ Shih-Chun Lee, MD, ${ }^{\mathrm{a}}$ \\ Ching Tzao, MD, PhD, ${ }^{\mathrm{a}}$ Jen-Chih Chen, $\mathrm{MD},{ }^{\mathrm{a}}$ and Hung Chang, $\mathrm{MD}, \mathrm{PhD}^{\mathrm{a}}$
}

Background: The crucial role of cigarette smoking in the development of pneumothorax is unclear because nonsmokers can also develop primary spontaneous pneumothorax. The purpose of this study was to clarify the pathophysiologic effects of cigarette smoking and its clinical correlations in primary spontaneous pneumothorax.

\begin{abstract}
Methods: Included were 115 specimens of lung tissue from patients with primary spontaneous pneumothorax who underwent video-assisted thoracoscopic surgery from January 2001 to December 2002. We reviewed the clinical features of 56 smokers and 59 nonsmokers with an average follow-up of 67 months. The pathologic findings of resected lung specimens were analyzed retrospectively.

Results: There were no statistical differences in sex, age, body height, body weight, body mass index, or the presence of blebs/bullae on computed tomography scans of the lung or under thoracoscopy between the 2 groups. In the smoking group, patients had more extensive respiratory bronchiolitis $(P<.001)$, a high prevalence of tobacco pigmentation $(P<.001)$, and a higher recurrence rate without or after surgery than the nonsmoking group $(57 \%$ vs $22 \%, P=.001$ and $8.9 \%$ vs $1.7 \%, P=.02$, respectively). Patients with extensive respiratory bronchiolitis had significantly higher nonoperative and postoperative recurrences than patients with nonextensive respiratory bronchiolitis $(P=.004$ and $P<.001$, respectively).
\end{abstract}

Conclusion: Cigarette smoking is associated with the pathophysiologic consequences of extensive respiratory bronchiolitis, which had a significant impact on the recurrence rates of primary spontaneous pneumothorax.

Primary spontaneous pneumothorax typically occurs in young, tall, lean men, especially smokers, with no obvious underlying lung diseases, and arguably results from the rupture of subpleural blebs or bullae. Primary spontaneous pneumothorax remains a significant health problem, with an annual incidence of 18 to 28 per 100,000 in the male population and 1.2 to 6.0 per 100,000 in the female population. ${ }^{1}$ The risk of primary spontaneous pneumothorax is linked to tobacco smoking and increases with length of exposure and daily consumption. ${ }^{2}$ Cigarette-induced pathologic changes in the small airways might contribute to the development of local emphysema with consequent formation of bullae. ${ }^{3}$ Questions remains about the clinical correlations between cigarette smoking and the occurrence of primary spontaneous pneumothorax. The aim of the present study was to clarify the influence of cigarette smoking and determine clinical

From the Division of Thoracic Surgery, Department of Surgery, ${ }^{a}$ Department of Pathology, ${ }^{\mathrm{b}}$ Tri-Service General Hospital, National Defense Medical Center, Taipei, Taiwan, ROC.

There is no substantial direct or indirect commercial financial incentive associated with publishing this article.

This research was supported by the Research Foundation of National Science Council (Grant No. NSC-95-2314-B-016-043), Taiwan, ROC.

Received for publication March 17, 2008; revisions received Oct 12, 2008; accepted for publication Dec 24, 2008.

Address for reprints: Yeung-Leung Cheng, MD, PhD, Division of Thoracic Surgery, Department of Surgery, Tri-Service General Hospital, 325, Section 2, Cheng-Kung

Road, Taipei 114, Taiwan, ROC (E-mail: ndmc0928@yahoo.com.tw).

J Thorac Cardiovasc Surg 2009;138:192-5

$0022-5223 / \$ 36.00$

Copyright (c) 2009 by The American Association for Thoracic Surgery doi:10.1016/j.jtcvs.2008.12.019 correlations in patients of primary spontaneous pneumothorax by examining resected specimens of the lung.

\section{PATIENTS AND METHODS}

A total of 115 lung tissue specimens from patients with primary spontaneous pneumothorax who underwent video-assisted thoracoscopic surgery (VATS) from January 2001 to December 2002 were selected via the CONSORT diagram (Figure 1). We reviewed the clinical features of 56 smokers and 59 nonsmokers with an average follow-up of 67 months. Data were collected for all patients and included a detailed history, number of episodes of pneumothorax and treatment modalities, age, sex, body height, body weight, body height/body weight ratio (centimeters/kilograms), body mass index (weight in kilograms divided by height in meters squared), and smoking habits. These patients initially received tube thoracostomies if indicated. All patients received high-resolution computed tomography (HRCT) scans of the chest. One radiologist, who was blinded to the clinical data, reviewed the HRCT of chest. The blebs/bullae were categorized into 3 groups according to the macroscopic findings under the thoracoscopy: type I, solitary and small bleb/bulla; type II, multiple and large blebs/bullae; and type III, diffuse and tiny blebs/bullae. ${ }^{4}$ Histologic sections of resection specimens were reviewed by 2 pathologists who were blinded to the clinical data. The specimens were embedded in paraffin. The blocks were cut in 1 to $2-\mu \mathrm{m}$ sections and stained. The pathologist assessed each specimen according to the following histologic features: the number of respiratory bronchioles, severity of respiratory bronchiolitis, pigment deposition, and arteriolar fibrosis. Severity of respiratory bronchiolitis was graded according to the extent of pigmented macrophage deposition around the respiratory bronchioles: Grade I (mild) was defined by the accumulation of pigmented macrophages limited to the lumen and wall of the respiratory and membranous bronchioles and to a single-layer ring of peribronchiolar alveoli; grade II (moderate) was defined by the extent of the deposition of tan-brown macrophages in the adjacent alveolar ducts and alveoli; grade III (extensive) was defined as massive deposition of pigmented macrophages in the peribronchiolar area, bronchiolar, and alveolar lumen. ${ }^{5}$ The definition of 


\section{Abbreviations and Acronyms}

HRCT $=$ high-resolution computed tomography

VATS $=$ video-assisted thoracoscopic surgery

microscopic emphysematous change of the resected lungs (panlobular, centrilobular, paraseptal, unclassified, blebs/bulla) and arterial intimal fibrosis is according to the textbook of Robbins and Cotran. ${ }^{6}$ Anthracosis is defined as black granular pigment. Hemosiderin is defined as golden yellow granular pigment. Tobacco pigment is defined as brown pigment accumulated in air spaces of macrophages.

Indications for surgical treatment of patients with primary spontaneous pneumothorax included persistent air leakage, tension pneumothorax at first presentation, hemopneumothorax, the occupation of the patient (eg, diver, pilot), and recurrences. A total of 115 patients underwent treatment by VATS with resection of the blebs/bullae and mechanical pleurodesis with gauze. We collected follow-up data via medical records, a telephone interview, and follow-up attendance at the outpatient department with an average of 67 months (range: 47-73 months).

\section{Statistical Analysis}

Descriptive data are expressed as mean \pm standard deviation. The Student $t$ test was used for continuous variables. The chi-square test was used for comparison of categoric variables between groups. The Statistical Package for the Social Sciences 11.0 software (SPSS Inc, Chicago, Ill) was used for all analyses.

\section{RESULTS}

A total of 115 patients with primary spontaneous pneumothorax underwent VATS were reviewed retrospectively. The clinical features of the patients were summarized and analyzed in Table 1. All patients presented initially with chest or back pain. Twenty-eight patients (24\%) presented with significant dyspnea, and 2 patients $(1.7 \%)$ had tachycardia and presented to the emergency department with cardiopulmonary distress. Indications for surgery of patients with primary spontaneous pneumothorax were persistent air leakage $(\mathrm{n}=11)$, tension pneumothorax at first presentation $(\mathrm{n}=2)$, hemopneumothorax $(n=4)$, simultaneous bilateral primary spontaneous pneumothorax $(n=3)$, patient occupation $(n=5)$, and recurrences $(n=45)$. Forty-five patients with a first episode of primary spontaneous pneumothorax underwent surgical intervention. There were no statistically significant differences in
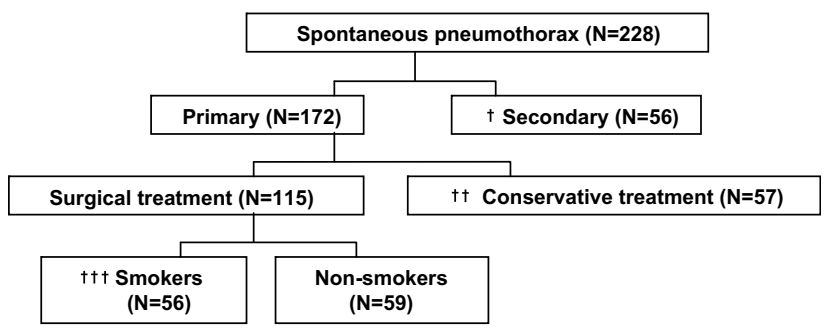

†COPD, asthma, cancer, pneumonia, lung abscess, et al

t† Observation, oxygen inhalation, needle aspiration, thoracostomy

$t \dagger+$ Active smokers or previous smokers

FIGURE 1. CONSORT diagram indicating the selection of study patients. $C O P D$, Chronic obstructive pulmonary disease.
TABLE 1. Comparisons of clinical features between smokers and nonsmokers among patients with primary spontaneous pneumothorax

\begin{tabular}{lccc}
\hline Variable & Smokers $(\mathbf{n = 5 6})$ & Nonsmokers $(\mathbf{n}=\mathbf{5 9})$ & $\boldsymbol{P}$ value* \\
\hline Sex & & & \\
$\quad$ Male & 54 & 54 & .478 \\
$\quad$ Female & 2 & 5 & \\
Age $(\mathrm{y})$ & $23.25 \pm 5.29$ & $21.76 \pm 5.39$ & .139 \\
BH $(\mathrm{cm})$ & $173.66 \pm 6.28$ & $174.33 \pm 6.80$ & .585 \\
BW $(\mathrm{kg})$ & $58.29 \pm 7.92$ & $59.24 \pm 7.54$ & .527 \\
BMI $\left(\mathrm{kg} / \mathrm{m}^{2}\right)$ & $19.27 \pm 2.13$ & $19.50 \pm 2.11$ & .576 \\
\hline
\end{tabular}

$B H$, Body height; $B W$, body weight; $B M I$, body mass index. *Significance level: $P<$ .05 (Student $t$ test).

age, body height, body weight, body mass index, or blebs/ bullae on computed tomography scans of the lung between the 2 groups (Table 2). Of the smoking patients, $80 \%$ had blebs/ bullae on HRCT, as did $80 \%$ of the nonsmokers $(P=.785)$. Eighty-three patients had blebs/bullae on the upper lobe; 13 patients had blebs/bullae in an additional lobe (non-upper lobe). Three patients had blebs/bullae only in the lower lobe. Of the smoking patients, $84 \%$ had blebs/bullae evident on thoracoscopic findings, as did $81 \%$ of the nonsmokers $(P=$ .924). Most of these patients had type II blebs/bullae (57\% of smokers, $56 \%$ of nonsmokers, respectively). Fifty-six of 115 patients $(49 \%)$ were smokers. There were no statistically

TABLE 2. Comparisons of categoric variables between smokers and nonsmokers among patients with primary spontaneous pneumothorax

\begin{tabular}{lccc}
\hline & \multicolumn{3}{c}{ Primary spontaneous pneumothorax } \\
\cline { 2 - 3 } & Smokers (56) & Nonsmokers (59) & $\boldsymbol{P}$ value* \\
\hline Blebs/bullae (HRCT) & & & .785 \\
Y & 45 & 47 & \\
N & 11 & 12 & \\
& &
\end{tabular}

Blebs/bullae

(thoracoscopy)

I
II
III

Bronchioles, no,

Respiratory bronchiolitis

I (mild)

II (moderate)

III (extensive)

Pigmentation

Tobacco

Anthracosis

Hemosiderin

Emphysema

Panlobular

Centrilobular

Paraseptal

Unclassified

Bullae

Arterial intimal fibrosis

Nonoperative recurrence

Postoperative recurrence

7
32
8

8
33
7

$4.9 \pm 3.6$

$3.6 \pm 3.9$

.065

HRCT, High-resolution computed tomography. ${ }^{*}$ Significance level: $P<.05$ ( $\mathrm{c}^{2}$ test) 
significant differences in the pathologic abnormalities seen in the resected specimen from the 2 groups except the occurrence of respiratory bronchiolitis and tobacco-related pigmentation. In the smoking group, all patients had pathologic findings of respiratory bronchiolitis in the resected specimens versus $49 \%$ of nonsmokers $(P<.001)$. In the smoking patients, tobacco-associated pigments occurred significantly more frequently than in nonsmokers $(66 \%$ of smokers vs $0 \%$ of nonsmokers; $P<.001$ ).

Forty-five patients presented with recurrence of primary spontaneous pneumothorax $(39 \%)$. The average time of occurrence after the first episode of pneumothorax was 17.7 months (range: 1-60 months). There was a higher prevalence of smokers among the patients presenting with a second pneumothorax $(57 \%$ vs $22 \% ; P=.001)$. All patients underwent VATS with resection of blebs/bullae and mechanical pleurodesis, with a mean follow-up period of 67 months (47-73 months). All patients recovered uneventfully except for 6 patients who had recurrence after operation (8.9\% of smokers vs $1.7 \%$ of nonsmokers, $P=.02$ ). There were no statistically significant differences in age, gender, or thoracoscopic findings between the nonextensive and extensive groups. Patients with extensive respiratory bronchiolitis had a higher recurrence rate after their first episode of primary spontaneous pneumothorax and higher postoperative recurrence (Table 3). These 6 patients recovered well after conservative treatment.

\section{DISCUSSION}

Spontaneous pneumothorax is a common condition that significantly affects health care expenditure. The average rate of recurrence is $30 \%$, with a range of $16 \%$ to $52 \%$. Many factors are involved in the pathogenesis of this

TABLE 3. Comparisons of severity of respiratory bronchiolitis between patients with and without recurrences

\begin{tabular}{lccc}
\hline \multirow{2}{*}{$\begin{array}{c}\text { Primary spontaneous } \\
\text { pneumothorax }\end{array}$} & \multicolumn{2}{c}{ Respiratory bronchiolitis } & \\
\cline { 2 - 3 } & Nonextensive & Extensive (III) & P value* \\
\hline Age (y) & $22 . \pm 5.0$ & $23 \pm 5.8$ & .782 \\
Gender & & & \\
$\quad$ Male (108) & 52 & 26 & .964 \\
$\quad$ Female (7) & 5 & 2 & \\
Thoracoscopic & 11 & & \\
I (15) & 46 & 19 & .572 \\
II (65) & 10 & 5 & \\
III (15) & & & \\
Nonoperative recurrence & 20 & 25 & \\
Y (45) & 67 & 3 & $.004 *$ \\
N (70) & & & \\
Postoperative recurrence & 1 & 5 & $<.001^{*}$ \\
Y (6) & 86 & 23 & \\
N (109) & & & \\
\hline
\end{tabular}

*Significance level: $P<.05$. Nonextensive: including no respiratory bronchiolitis, grade I respiratory bronchiolitis, and grade II respiratory bronchiolitis. Postoperative recurrence: recurrent pneumothorax after VATS. disease. The hazardous effects of smoking tobacco have been recognized. Cigarette smoke is a complex mixture of more than 4000 compounds and causes a variety of pulmonary and systemic effects in human. ${ }^{8,9}$ The associations among tobacco smoking, bronchogenic carcinoma, and obstructive airway and interstitial lung diseases have been widely reported. ${ }^{10,11}$ Cigarette smoking increases the relative risk of contracting a first pneumothorax approximately 9-fold in women and 22-fold in men. ${ }^{2}$ The trapping of distal air because of smoking-induced bronchiolar inflammation, leading to alveolar overdistension and rupture, may also be a contributing factor. ${ }^{7}$ Cottin and colleagues ${ }^{12}$ reported the high prevalence of respiratory bronchiolitis in patients with spontaneous pneumothorax. This small airway remolding represents a response to repeated inflammatory insults evoked by cigarette smoke or reflects a propensity to develop abnormal inflammatory reactions to minor stimuli. Cigarette smoke drives small-airway remodeling by induction of growth factors in the airway wall via increased release of active transforming growth factor- $\beta .{ }^{13}$ However, the pathophysiologic effects of cigarette smoking on the development of primary spontaneous pneumothorax and clinical correlation are not well established.

Controversy still exists around the occurrence of bullae and episodes of pneumothorax. This is partly because of differing views on the pathophysiology of primary spontaneous pneumothorax, especially on the causal role of the rupture of lung bullae. This debate has not only pathophysiologic implications but also important treatment implications. Air leakage occurring elsewhere on the visceral pleural surface independently of bullae or blebs has led to the concept of "pleural porosity" as a cause of primary spontaneous pneumothorax. ${ }^{14-16}$ Some authors have reported that computed tomography ${ }^{17}$ or thoracoscopic ${ }^{18}$ evidence of bullae during the evaluation of spontaneous pneumothorax is not predictive of recurrence. In this study, the incidence of blebs/bullae on thoracoscopic findings was $83 \%$ ( $84 \%$ of smokers and $81 \%$ of nonsmokers, $P=.924$ ), that is, no difference in the occurrence of blebs/bullae between the smokers and nonsmokers. This phenomenon encouraged us to determine the real pathophysiologic changes in primary spontaneous pneumothorax, especially in the cigarette-smoking patients.

Respiratory bronchiolitis is a common inflammatory lesion of respiratory bronchioles that occurs mainly with cigarette smoking. The histologic appearance is characterized by the accumulation of pigmented macrophages within respiratory bronchioles and surrounding airspaces with minimal associated mural inflammation. ${ }^{19}$ It is likely that this lesion occurs with highly variable severity in the lungs of all cigarette smokers and that the change may persist after smoking cessation. ${ }^{5}$ The prevalence of respiratory bronchiolitis in smokers was high $(98 \%) .{ }^{5}$ In our study, all the smoking patients had the pathologic findings of respiratory bronchiolitis in their resected specimen. In the nonsmokers, 
$49 \%$ had the pathologic abnormalities of respiratory bronchiolitis. We found that the smokers had a higher postoperative recurrence rate than the nonsmokers. Five of six patients with postoperative recurrence had extensive respiratory bronchiolitis. We speculated that cigarette smoking is associated with the pathophysiologic consequences of extensive respiratory bronchiolitis, which had a significant effect on recurrent primary spontaneous pneumothorax and postoperative recurrences.

Tobacco-induced chronic inflammatory reaction may spread centrifugally from the small airways to the adjacent parenchyma and lead to progressive destruction of peribronchiolar alveoli. ${ }^{20}$ Some support for this concept has come from the demonstration of a relationship between bronchiolar disease and centriacinar emphysema. ${ }^{21,22}$ In our study, the emphysematous lesions and patterns of emphysema showed no difference between smokers and nonsmokers. The association between tobacco-induced respiratory bronchiolitis and focal emphysema with consequent formation of blebs/bullae remains to be studied.

The rate of recurrence after the first episode of primary spontaneous pneumothorax was higher in smokers than in nonsmokers. However, cessation of smoking among patients after the first episode of primary spontaneous pneumothorax was not evaluated during the 73 months of follow-up. In addition, we did not evaluate the influence of the secondhand smoke in patients with primary spontaneous pneumothorax. More prospective studies are required to evaluate this issue.

Until now, there has been no clinical correlation between the pathophysiologic changes of cigarette smoking and the occurrence of primary spontaneous pneumothorax. In this study, we clarify the possible association between cigarette smoking and primary spontaneous pneumothorax.

\section{CONCLUSIONS}

In patients with primary spontaneous pneumothorax, cigarette smoking is associated with the pathophysiologic consequences of extensive respiratory bronchiolitis, which has a significant impact on the rate of recurrence of primary spontaneous pneumothorax.

\section{References}

1. Henry M, Arnold T, Harvey J. BTS guidelines for the management of spontaneous pneumothorax. Thorax. 2003;58(Suppl 2):39-52.

2. Bense L, Eklund G, Wiman LG. Smoking and the increased risk of contracting spontaneous pneumothorax. Chest. 1987;92:1009-12.

3. Weissberg D, Refaely Y. Pneumothorax. Experience with 1,199 patients. Chest 2000;117:1279-85.

4. Tamura M, Ohta Y, Sato H. Thoracoscopic appearance of bilateral spontaneous pneumothorax. Chest. 2003;124:2368-71.

5. Fraig M, Shreesha U, Savici D, et al. Respiratory bronchiolitis: a clinicopathological study in current smokers, ex-smokers, and never-smokers. Am J Surg Pathol. 2002;26:647-53.

6. Kumar V, Abbas AK, Fausto N, eds. Robbins and Cotran Pathologic Basis of Disease, 7th edition. Amsterdam: Elsevier; 2004. p. 717-22.

7. Schramel FM, Postmus PE, Vanderschueren RG. Current aspects of spontaneous pneumothorax. Eur Respir J. 1997;10:1372-9.

8. Bartecchi CE, Mackenzie TD, Schrier RW. The human costs of tobacco use. N Engl J Med. 1994;330:907-80.

9. Murin S, Hilbert J, Reilly SJ. Cigarette smoking and the lung. Clin Rev All Immunol. 1997;15:307-36.

10. Ryu JH, Colby TV, Hartman TE, et al. Smoking-related interstitial lung diseases: a concise review. Eur Respir J. 2001;17:122-32.

11. Desai SR, Ryan SM, Colby TV. Smoking-related interstitial lung diseases: histopathological and imaging perspectives. Clin Radiol. 2003;58: 259-68.

12. Cottin V, Streichenberger N, Gamondès J-P, et al. Respiratory bronchiolitis in smokers with spontaneous pneumothorax. Eur Respir J. 1998;12:702-4.

13. Churg A, Tai H, Coulthard T, et al. Cigarette smoke drives small airway remodeling by induction of growth factors in the airway wall. Am J Respir Crit Care Med. 2006;174:1327-34.

14. Ayed AK, Bazerbashi S, Ben-Nakhi M, et al. Risk factors of spontaneous pneumothorax in Kuwait. Med Princ Pract. 2006;15:338-42.

15. Masshoff W, Hofer W. Pathology of so-called idiopathic spontaneous pneumothorax. Dtsch Med Wochenschr. 1973;98:801-5.

16. Radomsky J, Becker HP, Hartel W. Pleural porosity in idiopathic spontaneous pneumothorax. Pneumologie. 1989;43:250-3.

17. Noppen M, Dekeukeleire T, Hanon S, et al. Fluorescein-enhanced autofluorescence thoracoscopy in patients with primary spontaneous pneumothorax and normal subjects. Am J Respir Crit Care Med. 2006;174:26-30.

18. Mitlehner W, Friedrich M, Dissmann W. Value of computed tomography in the detection of bullae and blebs in patients with primary spontaneous pneumothorax. Respiration. 1992;59:221-7.

19. Janssen JP, Schramel FM, Sutedja TG, et al. Videothoracoscopic appearance of first and recurrent pneumothorax. Chest. 1995;108:330-4.

20. Finkelstein RA, Cosio MG. Disease of the small airways in smokers: smokers' bronchiolitis. In: Epler GR, ed. Diseases of the Bronchioles. New York: Raven Press; 1994:115-37.

21. Kim WD, Eidelman DH, Izquierdo JL, et al. Centrilobular and panlobular emphysema in smokers: two distinct morphologic and functional entities. Am Rev Respir Dis. 1991;144:1385-90.

22. Willems LNA, Kramps JA, Stijnen T, et al. Relation between small airways disease and parenchymal destruction in surgical lung specimens. Thorax. 1990;45: 89-94. 